\title{
Contribution of the disk emission to the broad emission lines in AGNs: Two-component model ${ }^{\star}$
}

\author{
L. Č. Popović ${ }^{1,2,3}$, E. Mediavilla ${ }^{4}$, E. Bon ${ }^{1,3}$, and D. Ilić 5 \\ 1 Astronomical Observatory, Volgina 7, 11160 Belgrade, Serbia \\ e-mail: [lpopovic; ebon] @aob.bg.ac.yu \\ 2 Astrophysikalisches Institut Potsdam, An der Sternwarte 16, 14482 Potsdam, Germany (Alexander von Humboldt fellow) \\ e-mail: lpopovic@aip.de \\ 3 Isaac Newton Institute of Chile, Yugoslavia Branch, Yugoslavia \\ ${ }^{4}$ Instituto de Astrofísica de Canarias C/ Vía Làctea, s/n 38200 La Laguna, Tenerife, Spain \\ e-mail: emg@ll.iac.es \\ 5 Department of Astronomy, Faculty of Mathematics, University of Belgrade, Studentski trg 16, 11000 Belgrade, Serbia \\ e-mail: dilic@matf.bg.ac.yu
}

Received 2 October 2003 / Accepted 3 May 2004

\begin{abstract}
We present an investigation of the structure of the emission line region in a sample of 12 single-peaked Active Galactic Nuclei (AGNs). Using the high resolution $\mathrm{H} \beta$ and $\mathrm{H} \alpha$ line profiles observed with the Isaac Newton Telescope (La Palma) we study the substructure in the lines (such as shoulders or bumps) which can indicate a disk or disk-like emission in Broad Line Regions (BLRs). Applying Gaussian analysis we found that both kinds of emission regions, BLR and NLR, are complex. In this sample the narrow [OIII] lines are composites of two components; NLR1 which have random velocities from $\sim 200$ to $500 \mathrm{~km} \mathrm{~s}^{-1}$ and systematic velocities toward the blue from 20 to $350 \mathrm{~km} \mathrm{~s}^{-1}$, and NLR2 with smaller random velocities $\left(\sim 100-200 \mathrm{~km} \mathrm{~s}^{-1}\right)$ and a redshift corresponding to the cosmological one. The BLR also have complex structure and we apply a two-component model assuming that the line wings originate in a very broad line region (VBLR) and the line core in an intermediate line region (ILR). The VBLR is assumed to be an accretion disk and the ILR a spherical emission region. The model fits very well the $\mathrm{H} \alpha$ and $\mathrm{H} \beta$ line profiles of the AGNs.
\end{abstract}

Key words. galaxies: Seyfert - line: profiles - accretion, accretion disks

\section{Introduction}

The concept of a disk geometry in the Broad Line Region (BLR) is very attractive because the most widely accepted model for Active Galactic Nuclei (AGNs) includes a super massive black hole fed by an accretion disk. The detection and modeling of some double-peaked Balmer lines has supported this idea (Perez et al. 1988; Chen et al. 1989; Chen \& Halpern 1989; Eracleous \& Halpern 1994; RodríguezArdila et al. 1996; Storchi-Bergmann et al. 1997; Livio \& Xu 1997; Ho et al. 2000; Shields et al. 2000; Strateva et al. 2003; Storchi-Bergmann et al. 2003; Eracleous \& Halpern 2003). However, the fraction of AGNs clearly showing double-peaked profiles is small and statistically insignificant. On the one hand, the existence of double-peaked lines should not be required as a necessary condition for the existence of a disk geometry in BLRs. Even if the emission in a spectral line comes from a disk, the parameters of the disk (e.g. inclination) can be such that one observes single-peaked lines

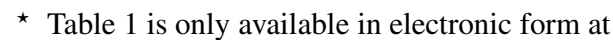
http://www . edpsciences.org
(Chen \& Halpern 1989; Dumont \& Collin-Souffrin 1990; Kollatschny \& Bischoff 2002; Kollatschny 2003). Also, a Keplerian disk with disk wind can produce single-peaked broad emission lines as normally seen in most of the AGNs (Murray \& Chiang 1997). On the other hand, taking into account the complexity of emission line regions of AGNs (see e.g. Sulentic et al. 2000), one might expect that the broad emission lines are composed of radiation from two or more kinematically and physically different emission regions, i.e. that multiple BLR emission components with fundamentally different velocity distributions are present (see e.g. Romano et al. 1996). Consequently, one possibility could be that the emission of the disk is masked by the emission of another emission line region. Recently, Popović (2003) investigated the physical processes in BLRs using a Boltzmann-plot method, and found that probably "physical conditions in regions which contribute to the line core and line wings are different". This supports the idea that the broad optical lines originate in more than one emission region, i.e. that the Broad Line Emission Region is complex and composed of at least two regions. Moreover, Corbin \& Boroson (1996) found that "the difference between the $\mathrm{Ly} \alpha$ and $\mathrm{H} \beta$ full 
width at zero intensity (FWZI) values provides additional evidence of an optically thin very broad line region (VBLR) lying inside an intermediate line region (ILR) producing the profile cores". Consequently we may expect that the VBLR can be formed in a disk or disk-like emission region. Moreover, recently Wang et al. (2003) investigated the central engines of 37 radio-loud QSOs and found that their accretion rates suggest that most of the objects possess standard optically thick, geometrically thin accretion disks. In fact, recently (Popović et al. 2001, 2002, 2003) it was shown that broad emission lines of at least three AGNs (Akn 120, NGC 3516 and III Zw 2) can be well fitted with a model which has two components: (i) an accretion disk and (ii) a region with a geometry different from a disk.

The aim of this paper is to test the validity of the twocomponent model of a BLR which contains an accretion disk and one additional emission region, i.e. to try to find evidence that suggests that the disk emission can contribute to the line emission even if the line profiles are single-peaked. To do this, we observed 12 AGNs in the $\mathrm{H} \alpha$ and $\mathrm{H} \beta$ wavelength region with the Isaac Newton Telescope (Sect. 2, throughout). High resolution spectra were analyzed first by Gaussian analysis (Sect. 3) and after that we applied the two-component model for BLRs assuming that it is composed of a VBLR and an ILR (Sect. 4).

\section{Observations and data reduction}

It is very important to notice that the two peaks produced by the disk may appear like two bumps in the blue and red parts of the $\mathrm{H} \beta$ and $\mathrm{H} \alpha$ line profiles. To find the substructure connected with disk emission one should obtain the spectral lines with a relatively high spectral resolution and $\mathrm{S} / \mathrm{N}$ ratio. We observed with the Isaac Newton Telescope (INT) 12 AGNs (see Table 1) which have been previously observed in the X-ray band (Fe K $\alpha$ line, see e.g. Nandra et al. 1997; Sulentic et al. 1998) and where, according to the X-ray results, one can expect that a disk geometry is present at least in the X-ray emitting region, i.e. that a disk exists whose signature might be observed in optical lines (emission of the outer part of the disk). The observed AGNs have no double-peaked $\mathrm{H} \alpha$ and $\mathrm{H} \beta$ lines.

The observations were performed with the $2.5 \mathrm{~m}$ INT at La Palma in the period of $21-25$ of January 2002. The Intermediate Dispersion Spectrograph (IDS) and the $235 \mathrm{cam}-$ era (with chip EEV10) in combination with the R1200Y (for the $\mathrm{H} \alpha$ wavelength region) and $\mathrm{R} 1200 \mathrm{~B}$ (for the $\mathrm{H} \beta$ wavelength region) gratings were used. The list of the observed AGNs with the coordinates, redshift, central wavelengths and the exposure times are given in Table 1. The seeing was around 1."1 and the slit width was $1^{\prime \prime}$. The spectral resolution was $\sim 1 \AA$. As one can see from Table 1, we observed the $\mathrm{H} \alpha$ and $\mathrm{H} \beta$ wavelength line region for all galaxies, except Mrk 141 where only the $\mathrm{H} \alpha$ region was observed. Also, after calibration of the spectra, the $\mathrm{H} \beta$ line of Mrk 493 was too weak and the red wing of the $3 \mathrm{C} 273 \mathrm{H} \alpha$ line was too noisy, so for these two spectra we use the low resolution spectra observed with the HST (on Sep. 4, 1996 and Jan. 31, 1999) with G400 and G750L gratings, respectively.
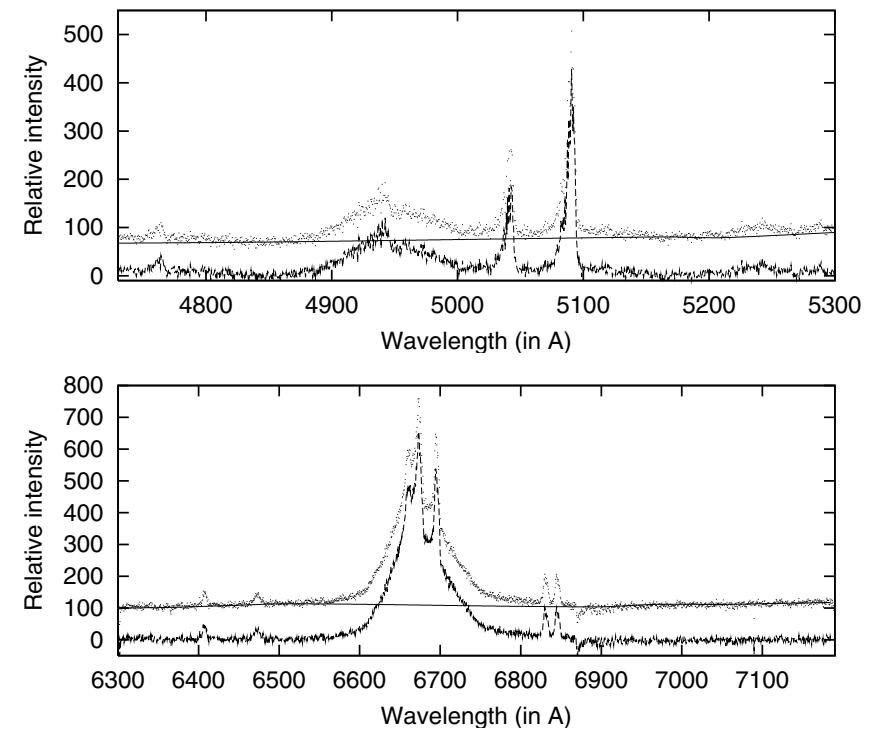

Fig. 1. The estimate of the continuum (solid line) in the case of Mrk $1040 \mathrm{H} \beta$ (top) and $\mathrm{H} \alpha$ (down). Dotted line the observed spectra and dashed line (below) the spectra after continuum subtraction.

$\mathrm{CuNe}$ and $\mathrm{CuAr}$ lamps were used for the wavelength calibration. Standard reduction procedures including flat-fielding, wavelength calibration, spectral response, and sky subtraction were performed with the help of the IRAF software package.

The software package DIPSO was used for reducing the level of the local continuum (by using the DIPSO routine "cdraw 1") fitted through the dots taken to be on the local continuum (see Fig. 1).

The redshifts of the considered AGNs were taken from Véron-Cetty \& Véron (2000).

\section{Line profile analysis}

To analyze the shape of the $\mathrm{H} \beta$ and $\mathrm{H} \alpha$ lines, we first fitted each line with the sum of Gaussian components. We used a $\chi^{2}$ minimalization routine to obtain the best fit parameters. We also assumed that the narrow emission lines can be represented by one or more Gaussian components. In the fitting procedure, we looked for the minimal number of Gaussian components needed to fit the lines. To limit the number of free parameters in the fit we set some a priori constraints (Popović et al. 2001, 2002, 2003):

- For the $\mathrm{H} \beta$ line in the fitting procedure we constrained the Gaussian parameters as follows:

(1) The three narrow Gaussians representing the $[\mathrm{OIII}] \lambda \lambda 4959,5007$ lines and the narrow $\mathrm{H} \beta$ component are fixed at the same redshift and the Gaussian widths are set proportional to their wavelengths. The Full Width at Half Maximum $(F W H M)$ is connected with the width $(W)$ of the Gaussian profile ${ }^{1}$ by $F W H M=2 W \sqrt{\ln 2}$;

(2) We imposed the intensity ratio of the two [OIII] $\lambda \lambda 49599,5007$ lines as 1:3.03 (Wiese et al. 1966).

(3) We included in the fit a red shelf Fe II template consisting of nine $\mathrm{Fe}$ II lines belonging to the multiplets 25,

\footnotetext{
${ }^{1}$ Given as $\exp \left[-(\Delta \lambda / W)^{2}\right]$.
} 

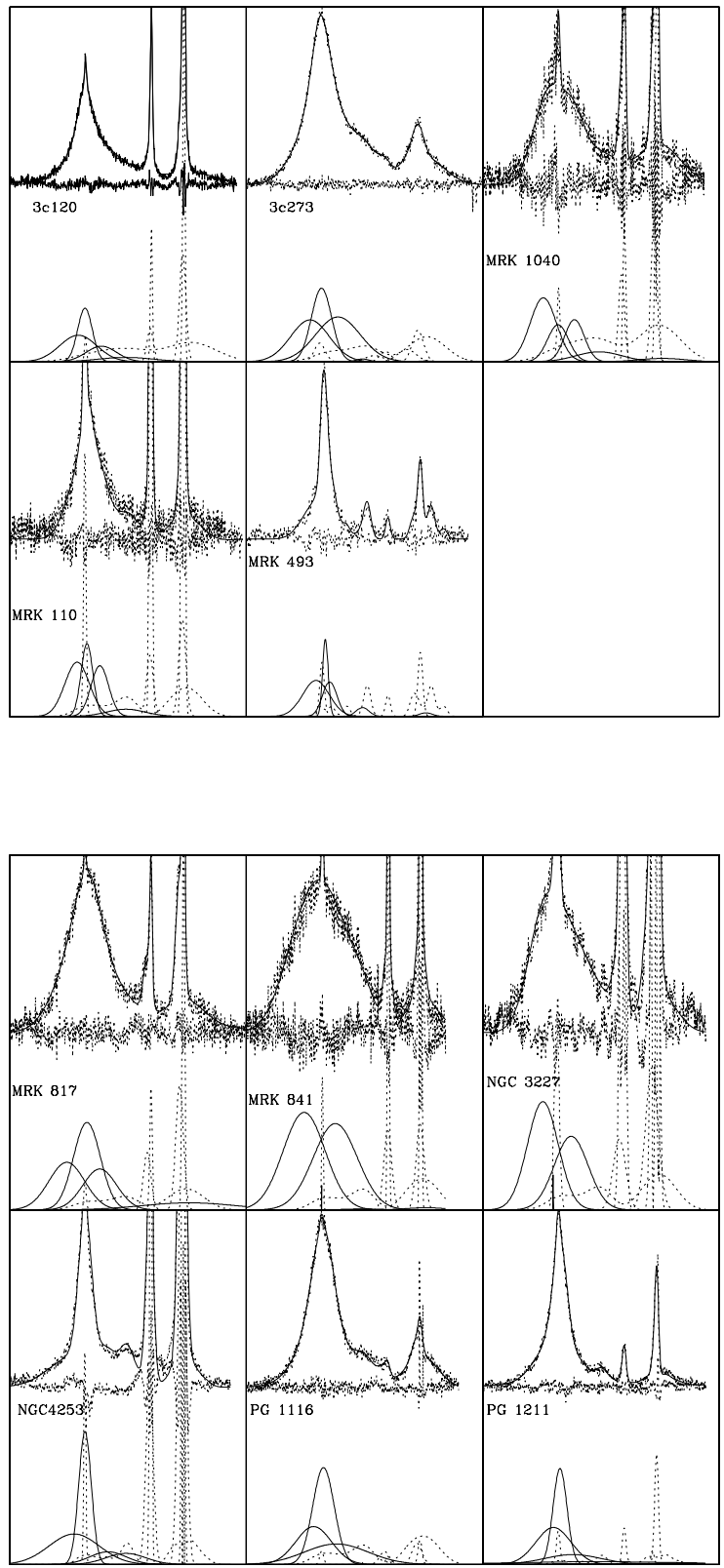

Fig. 2. Decomposition of the $\mathrm{H} \beta$ lines of the observed AGNs. The dashed lines represent the observations and the solid lines show the profile obtained by Gaussian decomposition. The Gaussian components are presented at the bottom. The dashed complex lines at the bottom represent the contribution of the Fe II, [OIII] and $\mathrm{H} \beta$ narrow lines.

36 and 42 (Korista 1992). We took the relative strength of these lines from Korista (1992) and assumed that all Fe II lines originate in the same region, that is, that all of them have the same redshift, and widths proportional to their wavelengths. The template contains $9 \mathrm{Fe}$ II lines from $4855.5 \AA$ to $5018.4 \AA$, and as one can see from Fig. 2 (e.g. Mrk $493 \mathrm{H} \beta$ ), provides a satisfactory fit to the iron lines in the $\mathrm{H} \beta$ wavelength region (Korista 1992; Popović et al. 2001, 2002, 2003). In comparison with the list of Fe II lines given by Sigut \& Pradhan (2003), the Fe II template used here gives a better fit to the Fe II lines of the present sample of AGNs.

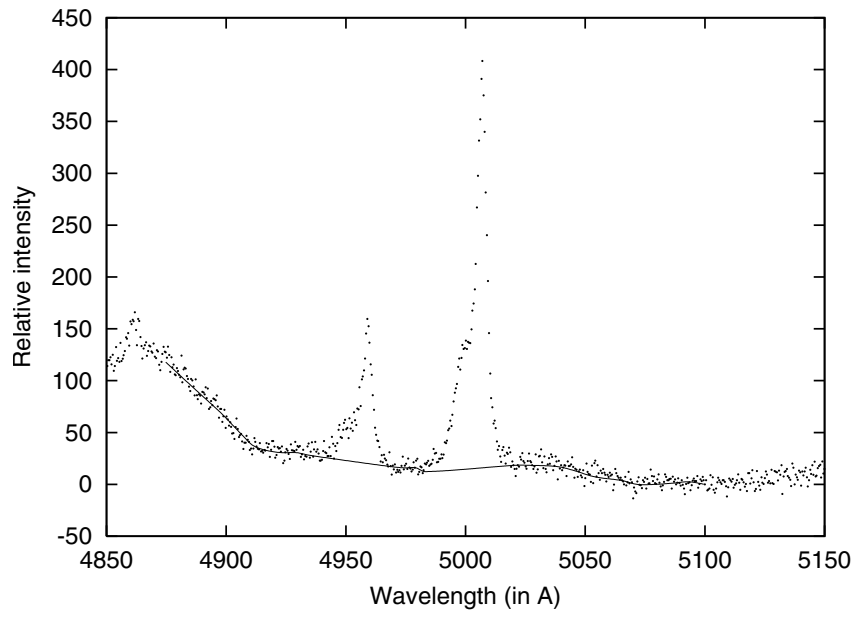

Fig. 3. The estimated contribution of the $\mathrm{H} \beta$ wing and other satellite lines in the $[\mathrm{OIII}] \lambda \lambda 4959,5007$ line wavelength region (solid line) for Mrk 817.

(4) We included the He I $\lambda \lambda 4921.93,5015.68 \AA$ A lines (Véron et al. 2002), assuming that they originate in the same region, i.e. that they have the same redshift and width. Here we should mention that the iron lines $4923.9 \AA$ and $5018.4 \AA$ (included in the iron template) are very close to these two He I lines. Therefore it is difficult to find the contribution of these components. We can obtain a satisfactory fit of the line profiles without them, but in the case of Mrk 1040, Mrk 110, Mrk 493, Mrk 817 and NGC 4253 the He I lines can contribute to the red wing of $\mathrm{H} \beta$ (see Fig. 2, full lines in the red wing of $\mathrm{H} \beta$ ), especially He I $\lambda 4923.9 \AA$, which tends to be stronger than He I $\lambda$ 5018.4 A.

After a first attempt to fit the lines in the $\mathrm{H} \beta$ wavelength region, we recognized that the [OIII] lines were well fitted only in the central part, but the wings could not be appropriately fitted with assumption (2), i.e. the [OIII] $\lambda \lambda 4959,5007$ lines show extended wings and cannot be properly fitted by only one Gaussian. We also notice that the wings are asymmetrical, with a shallower slope towards the blue.

To find an acceptable fit of the [OIII] lines we first included two more Gaussians which followed constraint (2). However, we found that a satisfactory fit in some of the AGNs can be obtained only if the intensity ratio of the $[\mathrm{OIII}] \lambda 5007$ and $[\mathrm{OIII}] \lambda 4959$ is left as a free parameter. Since our main purpose is to find the best fit in order to subtract the satellite and narrow lines from the $\mathrm{H} \beta$ and $\mathrm{H} \alpha$ line profile, we therefore decided to leave as a free parameter the intensity ratio of the blue-shifted gaussian components of the [OIII] $\lambda \lambda 4959,5007$ lines. As a rule we find that the second Gaussian of the [OIII] $\lambda \lambda 4959,5007$ lines is shifted to the blue and more broadened than the central narrow one (see Fig. 2). To check our results for the different components of the [OIII] lines, we subtracted the contributions of Fe II and $\mathrm{He} \mathrm{I}$ as well as the $\mathrm{H} \beta$ red wing from [OIII] using the DIPSO procedure for the continuum (see Fig. 3). Then we fitted the [OIII] lines using two Gaussians and the ratio of the [OIII] lines 1:3.03. In this case we can fit the [OIII] lines of 


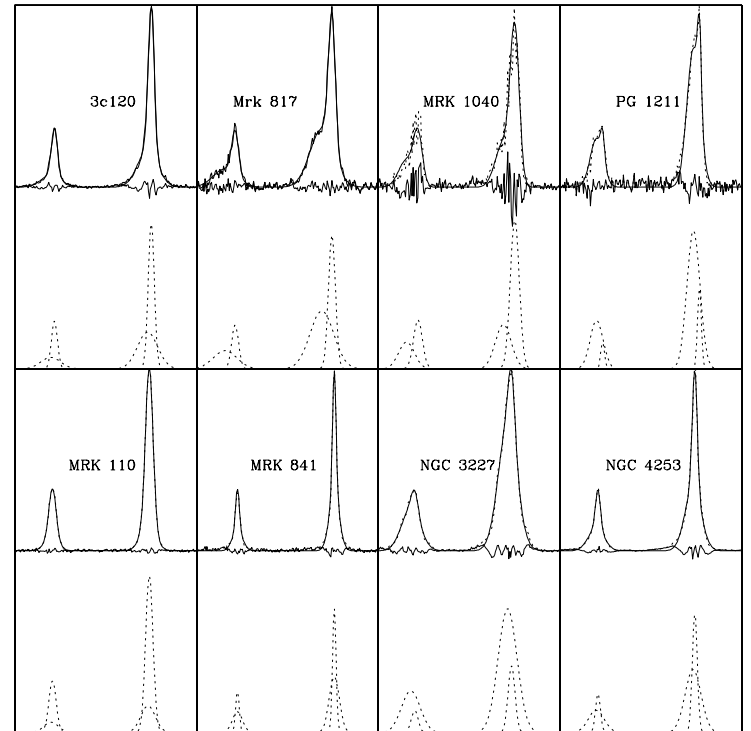

Fig. 4. Decomposition of the [OIII] lines.

Table 2. The parameters of the Gaussian components of the [OIII] lines. Gaussian widths (W) and $\Delta z=z_{\mathrm{NLR} 2}-z_{\mathrm{NLR} 1}$ are given in $\mathrm{km} \mathrm{s}^{-1}$. The intensity ratio $R_{\mathrm{NLR} 1}=I_{5007} / I_{4959}$ of the "blue component" obtained from the Gaussian fit, the measured flux ratio $R_{\mathrm{F}}=$ $F_{5007} / F_{4959}$ and central intensity ratio of the "blue" and "central" Gaussian components $\left(I_{\mathrm{B}} / I_{\mathrm{C}}\right)$ are also given (Cols. 2, 3 and 6$)$. The data for the objects denoted with stars should be taken with caution, because their $[\mathrm{OIII}]$ lines are too weak.

\begin{tabular}{ccccccc}
\hline \hline Object & $W_{\text {NLR1 }}$ & $R_{\text {NLR1 }}$ & $R_{\mathrm{F}}$ & $W_{\text {NLR2 }}$ & $\Delta z$ & $I_{\mathrm{B}} / I_{\mathrm{C}}$ \\
\hline 3c120 & 420 & 3.0 & $2.9 \pm 0.1$ & 130 & -110 & 0.25 \\
3c273* & 950 & 2.7 & - & 497 & -200 & 0.39 \\
Mrk 1040 & 330 & 1.8 & $2.4 \pm 0.1$ & 190 & -340 & 0.30 \\
Mrk 110 & 300 & 2.6 & $3.0 \pm 0.1$ & 150 & -40 & 0.17 \\
Mrk 817 & 510 & 3.0 & $3.0 \pm 0.1$ & 145 & -300 & 0.43 \\
Mrk 841 & 215 & 2.6 & $2.8 \pm 0.1$ & 80 & -20 & 0.45 \\
NGC 3227 & 400 & 3.0 & $3.0 \pm 0.1$ & 140 & -120 & 1.87 \\
NGC 4253 & 340 & 2.8 & $3.0 \pm 0.1$ & 105 & -55 & 0.55 \\
PG 1116* & 650 & 1.8 & - & 239 & -345 & 0.88 \\
PG 1211 & 270 & 2.8 & $3.0 \pm 0.1$ & 81 & -180 & 1.8 \\
\hline
\end{tabular}

some of the AGNs in an adequate way (e.g. the [OIII] lines of 3C 120, Mrk 817, NGC 3227). There were small differences in the residue for Mrk 110, NGC 4253 and PG 1211. But for the remaining two AGNs (Mrk 1040 and Mrk 841) we could obtain a satisfactory fit only with the intensity ratio of [OIII] $\lambda 5007$ and [OIII] $\lambda 4959$ left as a free parameter (see Fig. 4). Indeed, we have measured the fluxes of the [OIII] lines and found that in these two AGNs the intensity ratio tends to be smaller than 3.03. The results of the Gaussian fitting analysis for the $[\mathrm{OIII}] \lambda \lambda 4959,5007$ lines are presented in Fig. 4, and in Table 2.

- For the $\mathrm{H} \alpha$ line, we assumed that $[\mathrm{NII}] \lambda \lambda 6548,6583$ and the $\mathrm{H} \alpha$ narrow component have the same redshift, and Gaussian widths proportional to their wavelengths. Taking into account that the two [NII] $\lambda \lambda 6548,6583$ lines belong to a transition within the same multiplet we assume an intensity ratio of 1:2.96 (see e.g. Wiese 1966). The fits of the $\mathrm{H} \alpha$ lines are
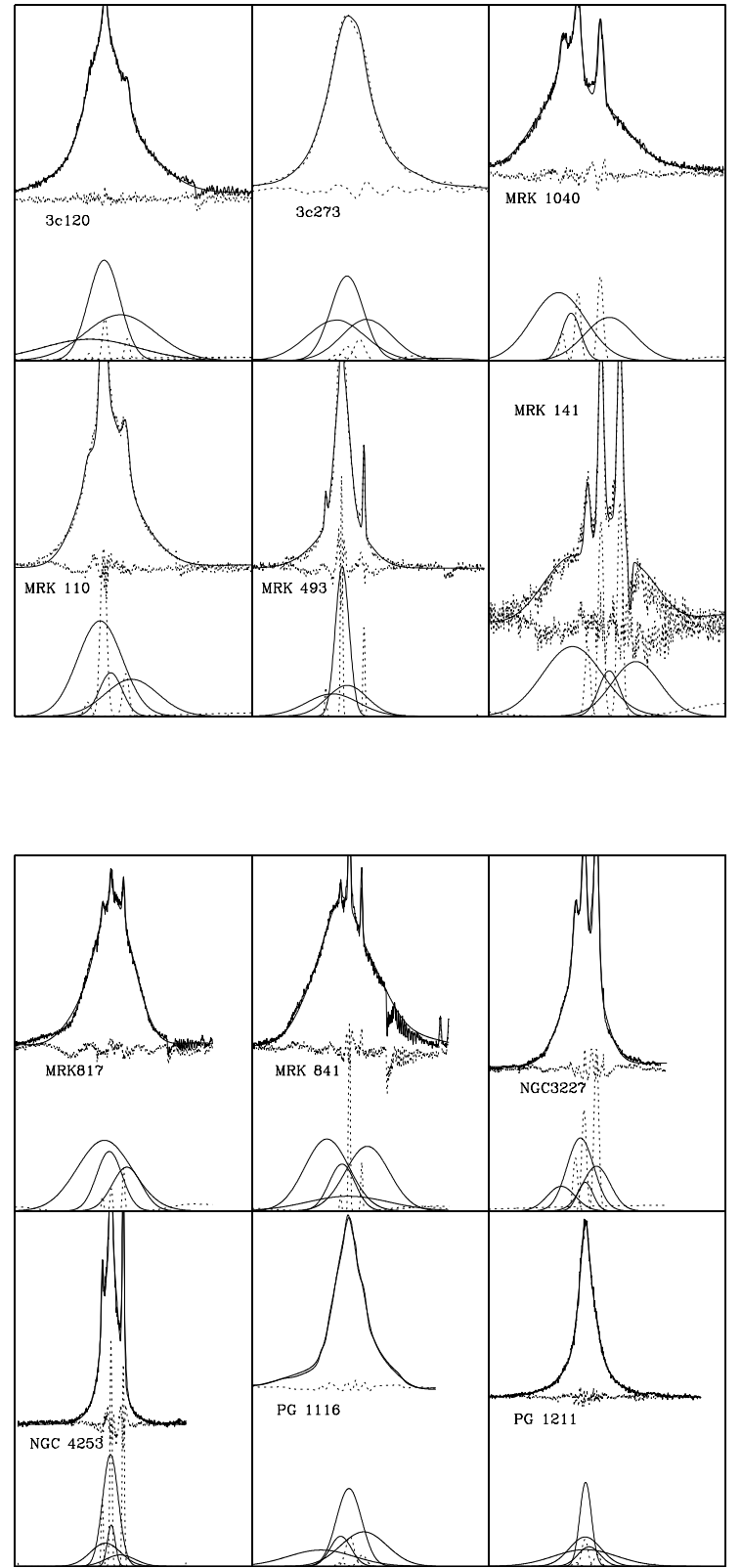

Fig. 5. The same as in Fig. 2. but for the $\mathrm{H} \alpha$ line. The narrow dashed lines at the bottom correspond to the narrow $\mathrm{H} \alpha$ and [NII] lines, the broad dashed lines correspond to the Fe II and He I contribution.

presented in Fig. 5. One can expect that other narrow lines in the optical spectra have the same shape as the [OIII] ones, but as the $[\mathrm{NII}] \lambda \lambda 6548,6583$ lines are heavily blended we are not able to resolve their fine structure. Hence, each of them has been fitted by only one Gaussian. On the other hand, we include the He I $\lambda 6678.15 \AA$ and SiII $\lambda 6371$ lines (Véron et al. 2002), and each line has been represented by one Gaussian. Also, one can expect a contribution of Fe II lines in the $\mathrm{H} \alpha$ wavelength region, but on the bases of the calculation given by Sigut $\&$ Pradhan (2003) there are no Fe II lines ${ }^{2}$ that can significantly contribute to the $\mathrm{H} \alpha$ line profile. Even if any lines are present their intensity is probably negligible. It has been shown (e.g.

2 The nearest lines to $\mathrm{H} \alpha$ are Fe II $\lambda 6456.385$ (near the blue wing) and Fe II $\lambda 7308.065$ (near the red wing). 


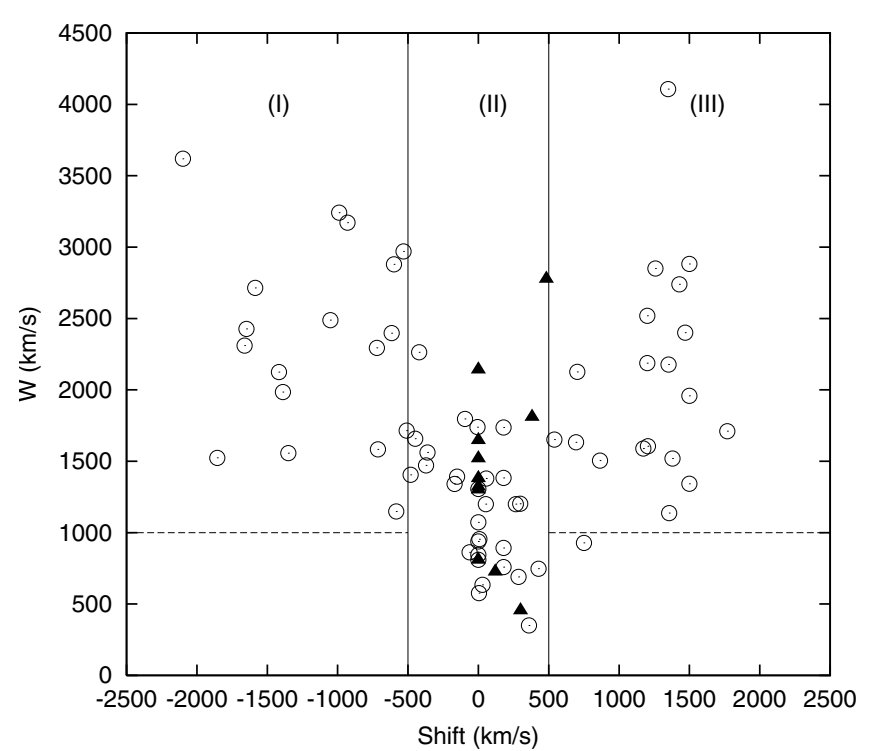

Fig. 6. The widths $(w)$ as a function of the inner shifts of the broad Gaussian components obtained for the $\mathrm{H} \alpha$ and $\mathrm{H} \beta$ lines of our sample of AGNs (open circles) and the Fe II template (full triangles).

Halpern \& Oke 1987; Lípari et al. 1993) that AGNs with strong iron emission in the $\mathrm{H} \beta$ wavelength region have a weak emission of Fe II in the $\mathrm{H} \alpha$ band (see e.g. Figs. 1 and 2 in Halpern \& Oke 1987). By inspection of the NIST database ${ }^{3}$ in the $\mathrm{H} \alpha$ region we found that a possible $\mathrm{Fe}$ II contribution may come from the permitted lines $3 \mathrm{~d}^{72} \mathrm{G}-3 \mathrm{~d}^{6}\left({ }^{3} \mathrm{G}\right) 4 \mathrm{~s}^{2} \mathrm{G}$ (Fe II lines $6700.64 \AA$ and $6873.84 \AA$ ) and $3 d^{7}{ }^{2} \mathrm{D} 2-3 d^{6}\left({ }^{3} \mathrm{D}\right) 4 \mathrm{~s}^{2} \mathrm{D}$ (lines $6746.53 \AA$, $6689.41 \AA$ and $6404.615 \AA$ ) as well as from FeII $\lambda$ 6456.387. In the fitting procedure we included these lines assuming that they originate from the same region, i.e. that they have the same width and redshift. As one can see from Fig. 5 (broad dashed lines), these lines, as well as the He I and SiII lines, make a negligible contribution to the $\mathrm{H} \alpha$ line profile, i.e. a satisfactory fit can be obtained without them.

\subsection{Discussion of the multi-Gaussian analysis}

In Fig. 6 we present the Gaussian widths of the different broad components versus their centroid velocities (relative to the systemic one). The different components appear relatively well separated in this diagram, showing the consistency of the multiGaussian decomposition. By inspection of Figs. 2, 4 and 5 as well as of the diagrams in Figs. 6 and 7, we can derive a number of conclusions concerning the broad line and the narrow line components, consequently concerning the BLR and the NLR.

\subsubsection{The broad emission lines}

Considering only the broad line Gaussian components we can conclude:

(i) that the $\mathrm{H} \beta$ and $\mathrm{H} \alpha$ line shapes of the considered AGNs are very complex, and usually cannot be described by one

\footnotetext{
${ }^{3}$ http://physics.nist.gov/PhysRefData/ contents-atomic.html
}

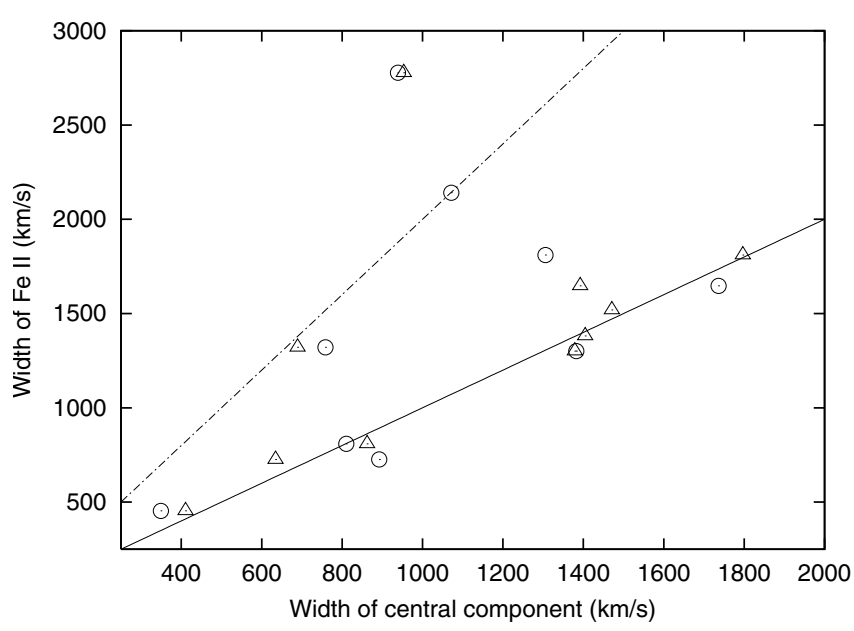

Fig. 7. The Gaussian width of the Fe II template $\left(w_{\text {FeII }}\right)$ as a function of the central broad Gaussian component width $\left(w_{\mathrm{C}}\right)$ of the $\mathrm{H} \alpha$ (open triangles) and $\mathrm{H} \beta$ (open circles) line. The solid line corresponds to $w_{\text {FeII }}=w_{\mathrm{C}}$ and the dashed one to $w_{\text {FeII }}=2 \cdot w_{\mathrm{C}}$. The points at the top, outside of the region between the two lines belong to $3 \mathrm{C} 273$.

Gaussian, i.e. the Gaussian decomposition indicates a very complex kinematical structure of the BLRs;

(ii) that the Gaussian decomposition indicates the existence of a central broad component (part (II) of Fig. 6) with low random velocities (from 500 to $1500 \mathrm{~km} \mathrm{~s}^{-1}$ ), and a redshift consistent with the systemic velocity (between $\pm 500 \mathrm{~km} \mathrm{~s}^{-1}$ )

(iii) that the red- and blue-shifted broad components (part (I) and (III) in Fig. 6) are often present in the fit. They tend to have higher random velocities with higher (positive or negative) shift. Such a near-symmetrical distribution of the broad red- and blue-shifted components may indicate that in the considered AGN sample a fraction of the emission, at least the wings, originates in an accretion disk;

(iv) the widths of the Fe II lines have different random velocities (Fig. 6) and a redshift consistent with the systemic velocity $\left( \pm 500 \mathrm{~km} \mathrm{~s}^{-1}\right)$. On the other hand, the Fe II random velocity $\left(w_{\mathrm{FeII}}\right)$ tends to vary linearly with the central broad component random velocity ( $w_{\mathrm{c}}$, see Fig. 7), with $w_{\mathrm{c}} \leq w_{\text {FeII }} \leq 2 w_{\mathrm{c}}$ except in the case of 3C 273 (the two points outside the region between these lines in Fig. 7). This may indicate that a significant fraction of the Fe II emission is created in the region emitting the core of the $\mathrm{H} \alpha$ and $\mathrm{H} \beta$ lines.

\subsubsection{The narrow emission lines}

The NLRs of the AGNs considered also show a complex structure, and we can clearly see at least two NLR regions:

(i) the NLR1, which has an internal random velocity from $\sim 200$ to $500 \mathrm{~km} \mathrm{~s}^{-1}$, and relative approaching velocities from 20 to $350 \mathrm{~km} \mathrm{~s}^{-1}$ with respect to the systemic redshift of the observed galaxy; and

(ii) the NLR2 which has an internal random velocity between $100-250 \mathrm{~km} \mathrm{~s}^{-1}$, and a redshift equal to the systemic one of the corresponding object. 
On the other hand, the intensity ratio of the components from the NLR2 follows the line ratio $(I(5007): I(4959)=3.03)$, while for the NLR1 in 2 AGNs, PG 1116 and Mrk 1040, this ratio is significantly smaller than 3.03 (see Table 2). Although a slight difference between the observed and predicted intensity ratio of the [OIII] lines might exist (Storey \& Zeippen 2000), this is certainly due to the bad S/N for PG 1116, and in the case of Mrk 1040, to the high residues of the fit (see Figs. 2 and 3).

The difference in shifts and widths of [OIII] $\lambda \lambda 4959,5007$ between these two NLRs indicate different kinematical and physical properties. Here we note that the parameters for the NLR1 of 3C 273 and PG $1116+215$ given in Table 2 should be taken with caution because of the low intensity of the [OIII] lines. We excluded these data in the discussion above.

The clear tendency of the NLR1 to have a blue-shifted systemic velocity (though in the case of Mrk 110 and Mrk 841 the blue-shift is marginal and very close to the cosmological redshift) supports the idea of a jet geometry of the NLR (see e.g. Dopita et al. 2003). In this case the receding jet component in the [OIII] lines might be obscured or absorbed by the host galaxy, so one sees only the outflowing gas from the closer part of the jet. Many papers have been devoted to the radial velocity difference between narrow and broad lines (starting e.g. from Gaskell 1982), especially the velocity difference between the [OIII] lines and $\mathrm{H} \beta$ that indicates a jet geometry of the NLR or of part of the NLR (see e.g. Bennert 2002; Zamanov 2002). On the other hand, 2D spectroscopy clearly shows that AGN NLRs have a complex structure (see e.g. Arribas 1997). The tendency for the [OIII] lines to be blue-shifted relative to the $\mathrm{H} \beta$ suggests that they are associated with a high-ionization outflow originating in these highly accreting sources (see e.g. Zamanov 2002).

\section{Two-component model for BLR}

Here we apply the two-component model in modeling the broad line shapes of 12 AGNs, where one component is the disk or disk-like region. We start from the paper of Corbin \& Boroson (1996), who investigated the combined ultraviolet and optical spectra of 48 QSOs and Seyfert 1 galaxies in the redshift range $0.034-0.774$. They found a statistically significant difference between the FWZI distributions of the Ly $\alpha$ and $\mathrm{H} \beta$ lines. The difference between the Ly $\alpha$ and $\mathrm{H} \beta$ FWZI values provides additional evidence for an optically thin VBLR (which might be a disk or disk-like region) which contributes to the line wings. It is located inside an ILR which produces the profile cores. Also, they found relative weakness of the correlations between the UV profile asymmetries and widths and those of $\mathrm{H} \beta$ line. This suggests a stratified structure of the BLR, consistent with the variability studies of Seyfert 1 galaxies (see e.g. Kollatschny 2003). The smaller average FWHM values of the UV lines compared to $\mathrm{H} \beta$ indicate that the ILR emission make a higher contribution to the UV lines, whereas in the Balmer lines the VBLR component is more dominant. This is also the case in well known AGNs with double-peaked Balmer lines, which usually show a single-peaked Ly $\alpha$ line (see e.g. the case of Arp 102B, Halpern et al. 1996).

The wings of the broad $\mathrm{H} \alpha$ emission line in the spectra of a large sample of AGNs (around 100 spectra) were investigated by Romano et al. (1996). They found an indication of multiple BLR emission. Moreover, recently Popović (2003) investigated the physical conditions in BLRs finding an indication that BLRs are complex and that physical conditions of the regions which contribute to the line core and the line wings are different.

Though a two-component model can probably also be represented by other geometries, we choose the one with: a disk giving the wings of the lines, and a spherical component giving the core of the lines.

\subsection{Theoretical point}

For the disk we use the Keplerian relativistic model of Chen \& Halpern (1989). The emissivity of the disk as a function of radius, $R$, is given by $\epsilon=\epsilon_{0} R^{-p}$. Generally, when trying to fit the double-peaked line profiles by disk emission one should leave this index as a free parameter. However, we have to take into account two facts: (1) we have here single-peaked lines, i.e. the profile coming from the disk is not a priori well defined; (2) we will be using a two-component model which includes more parameters than a disk-only model. We should therefore include some constraints. Since the illumination is due to a point source radiating isotropically, located at the center of the disk, the flux in the outer disk at different radii should vary as $r^{-3}$ (Eracleous $\&$ Halpern 1994). We note here that this is indeed the way the incident flux varies, but not necessarily the way in which the lines respond to it (Dumont \& Collin-Souffrin 1990; Rokaki \& Boisson 1999; Eracleous \& Halpern 2003). However, the power index $p \approx 3$ can be adopted as a reasonable prescription at least for $\mathrm{H} \alpha$ (Eracleous \& Halpern 2003). In our case, as one can see in Fig. 8, the shapes of $\mathrm{H} \alpha$ and $\mathrm{H} \beta$ in our sample are practically the same, so we can fit an averaged profile (see Sect. 4.2). Therefore we will start our fitting procedure by imposing the constraint $p=3$, but later we will change this parameter and use other constraints (see Sect. 4.2).

We express the disk dimension in gravitational radii $\left(R_{\mathrm{g}}=\right.$ $G M / c^{2}, G$ being the gravitational constant, $M$ the mass of the central black hole, and $c$ the velocity of light). The local broadening parameter $(\sigma)$ and shift $\left(z_{1}\right)$ within the disk have been taken into account as in Chen \& Halpern (1989), i.e. the $\delta$ function has been replaced by a Gaussian function:

$\delta \rightarrow \exp \frac{\left(\lambda-\lambda_{0}-z_{1}\right)^{2}}{2 \sigma^{2}}$

where $z_{1}$ and $\sigma$ are the local shift and the broadening parameter of the disk emission, respectively.

On the other hand, we assume that the kinematics of the additional emission region can be described as the emission of a spherical region with an isotropic velocity distribution, i.e. with a local broadening $w_{\mathrm{G}}$ and shift $z_{\mathrm{G}}$. Consequently, the emission line profile can be described by a Gaussian function. The whole line profile can be described by the relation:

$I(\lambda)=I_{\mathrm{AD}}(\lambda)+I_{\mathrm{G}}(\lambda)$

where $I_{\mathrm{AD}}(\lambda), I_{\mathrm{G}}(\lambda)$ are the emissions of the relativistic accretion disk and of an additional region, respectively. 


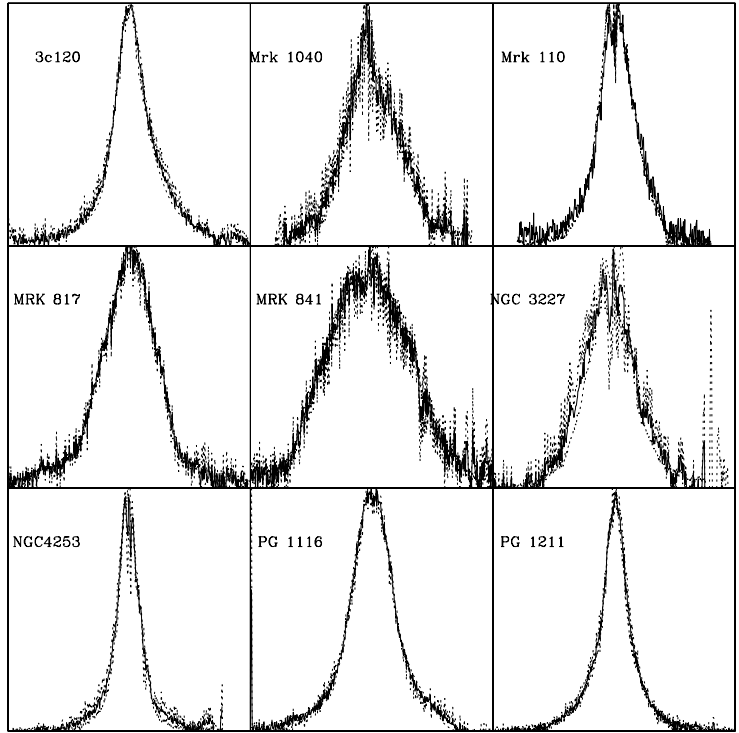

Fig. 8. The comparison of $\mathrm{H} \beta$ and $\mathrm{H} \alpha$ (dashed lines) profiles with an averaged one (solid line).

\subsection{Fitting procedure}

Before performing the fitting we "cleaned" the spectra by subtracting (i) from $\mathrm{H} \beta$ the narrow $\mathrm{H} \beta$ line, the narrow [OIII] lines, the He I lines, and the Fe II template; (ii) from $\mathrm{H} \alpha$ the narrow $\mathrm{H} \alpha$ and $[\mathrm{NII}]$ lines. Furthermore, we normalized the intensities of $\mathrm{H} \alpha$ and $\mathrm{H} \beta$ to unity and converted the wavelength into a velocity scale: $\lambda \rightarrow X=\left(\lambda-\lambda_{0}\right) / \lambda_{0}$. These conversions allowed us to compare the $\mathrm{H} \alpha$ and $\mathrm{H} \beta$ high resolution profile. We found that in the AGNs of the sample the $\mathrm{H} \alpha$ and $\mathrm{H} \beta$ have similar profiles (see Fig. 8). That concept supports the case that both lines are formed in the same emission region. First we found an averaged line profile from the $\mathrm{H} \alpha$ and $\mathrm{H} \beta$ lines from each AGN (here we should note that for 3C 273 only the high resolution $\mathrm{H} \beta$, and for Mrk 493 and Mrk 141 only $\mathrm{H} \alpha$ were used). The averaged profile for each AGN was fitted with the above described two-component model.

When a chi-square minimization including all the parameters was attempted, it was found that the results are very dependent on the initial values given to the parameters. As mentioned above, the reason for this is that we apply a two-component model to single-peaked lines, so the number of free parameters is too large. To overcome this problem we have to use the additional constraint that the disc component fits the line wings, and the spherical component the line core. With this aim, we tried several values for the inclination. The starting point for the inclination can be the relative displacement of redand blue-shifted Gaussians, $\Delta z$. We use the empirical relation given by Smak (1981) (see also Popović et al. 2002, 2003). If we assume that a disk (or a disk-like) region exists, we can roughly estimate the parameters of the disk using the results of Gaussian analysis and the relationship (see Popović et al. 2002 for more details) $\sin i \approx \Delta z \sqrt{2 \cdot R_{\mathrm{out}}}$, where $i$ is the inclination of the disk, and $R_{\text {out }}$ is the outer radius given in gravitational radii. Taking into account that $\sin i \leq 1$, we can estimate the maximal outer radius. From Fig. 6 one can estimate that $\sim 0.00033\left(\sim 1000 \mathrm{~km} \mathrm{~s}^{-1}\right) \leq \Delta z \leq 0.013\left(\sim 4000 \mathrm{~km} \mathrm{~s}^{-1}\right)$, thus
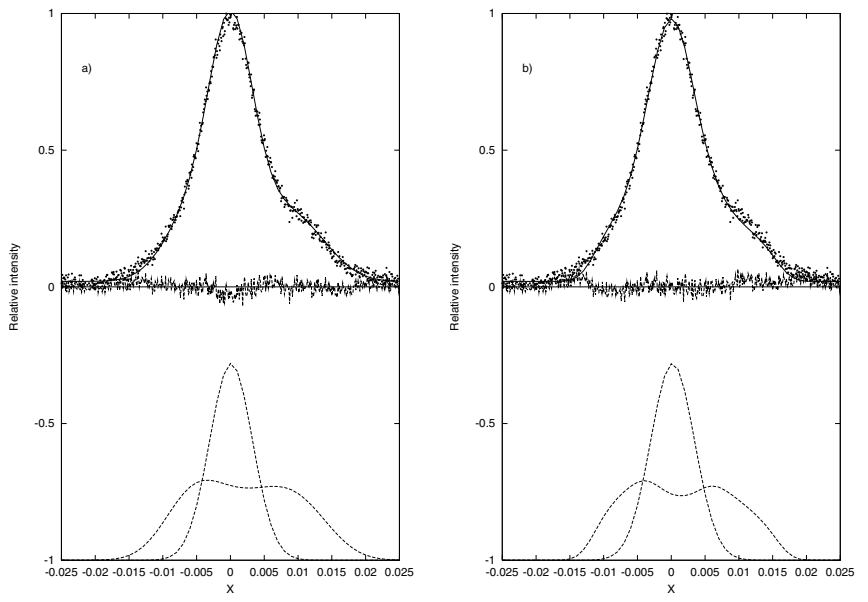

Fig. 9. Two fits of 3C 273 with the two-component model. The disk parameters are: a) $i=14^{\circ}, R_{\mathrm{inn}}=400 R_{\mathrm{g}}, R_{\mathrm{out}}=1420 R_{\mathrm{g}}, W_{\mathrm{d}}=$ $1620 \mathrm{~km} \mathrm{~s}^{-1}, p=3.0\left(W_{\mathrm{G}}=1350 \mathrm{~km} \mathrm{~s}^{-1}\right)$; b $) i=29^{\circ}, R_{\mathrm{inn}}=1250 R_{\mathrm{g}}$, $R_{\text {out }}=15000 R_{\mathrm{g}}, W_{\mathrm{d}}=700 \mathrm{~km} \mathrm{~s}^{-1}, p=2.8\left(W_{\mathrm{G}}=1380 \mathrm{~km} \mathrm{~s}^{-1}\right)$.

the maximal outer radius may be in the interval $\sim$ a few $10^{3} R_{\mathrm{g}}$ to $\sim$ a few $10^{5} R_{\mathrm{g}}$ for the highest inclination. On the other hand, from previous investigations (Chen et al. 1989; Eracleous \& Halpern 1994; Eracleous 2003) it was found that the outer radius of the disk has typical dimensions of $\sim$ a few $10^{3} R_{\mathrm{g}}$; in that case, the inclination for the sample is in the interval $i \sim 5^{\circ}-25^{\circ}$.

As we noted in Sect. 4.1, first we fixed $p=3$, and chose different values of the inclination. Then we found the best fit by eye and after that we used a "fixed" inclination such that other parameters can change more (with greater step) than the inclination ${ }^{4}$. After that we performed a chi-square fitting of the parameters starting from suitable initial values. Note that this is not an "orthodox" chi-square fitting procedure, but in this case the main point is not only to find the best fit, as we did with the Gaussian fitting procedure, but also to try to explain the line profile with our two-component model. Consequently, we found that the fit of the wings of the BELs strongly restricts the value of the inner radius and the "local" broadening, i.e. the random velocity of emission gas in the disk. We found that the inclinations of the disk are relatively small, between $5^{\circ}$ and $15^{\circ}$. One should have expected higher inclinations in the sample (e.g. on average $30^{\circ}$ ).

Therefore we decided to choose the inclination in the range $i \geq 25^{\circ}$, leaving $p$ as a free parameter, and looking for the maximal inclinations. We obtained reasonably good fits for the lines of more than half of the sample, for $p<3$ and higher values of the inner and outer accretion disk radii (see Figs. 9a,b). Note that low values of the emissivity index, $p$, were obtained also by Eracleous \& Halpern (1994) and Eracleous (2003) when fitting double peaked lines. Consequently, we did fitting tests for different fixed values of $p$ in the range from 1.0 to 3.0. Also, in this case we can find acceptable fit for some AGNs. As an example, in Fig. 9 one can see two fits of the 3C 273 line with different parameters. The best fit corresponds to Fig. 9a, but the

\footnotetext{
4 We use the SIMPLEX method for fitting that allows us to define the different magnitudes of "reflections" (the steps to change the parameters).
} 
Table 3. The parameters of the disk: $z_{1}$ is the shift and $W_{1}=\sqrt{2} \sigma$ is the Gaussian broadening term from the disk which is a measure of the random velocity in the disk, $R_{\text {inn }}$ is the inner radius, $R_{\text {out }}$ is the outer radius. $z_{\mathrm{G}}$ and $W_{\mathrm{G}}$ represent the parameters of the Gaussian component.

\begin{tabular}{|c|c|c|c|c|c|c|c|c|}
\hline Object & $i$ & $z_{1}^{\min , \max }$ & $W_{1}^{\min , \max }\left(\mathrm{km} \mathrm{s}^{-1}\right)$ & $R_{\mathrm{inn}}^{\min }\left(R_{\mathrm{g}}\right)$ & $R_{\text {out }}^{\max }\left(R_{\mathrm{g}}\right)$ & $z_{\mathrm{G}}^{\min , \max }$ & $W_{\mathrm{G}}\left(\mathrm{km} \mathrm{s}^{-1}\right)$ & $p^{\min }$ \\
\hline $3 \mathrm{C} 120$ & $8-30$ & $-300,+300$ & 1050,1500 & 350 & 20000 & $+30,+300$ & $900 \pm 150$ & 2.0 \\
\hline $3 \mathrm{C} 273$ & $12-30<$ & $-30,+300$ & 690,1760 & 400 & 15400 & $+30,+60$ & $1380 \pm 150$ & 2.3 \\
\hline MRK 1040 & $5-27<$ & $-250,+300$ & 800,1400 & 100 & 18000 & $0 \pm 30$ & $500 \pm 200$ & 1.3 \\
\hline MRK 110 & $7-50$ & $-320,+300$ & 450,1250 & 400 & 49000 & $+150 \pm 30$ & $960 \pm 50$ & 1.7 \\
\hline MRK 141 & $12-33$ & $-630,-450$ & 700,1500 & 300 & 10000 & $+200,+300$ & $1620 \pm 100$ & 2.1 \\
\hline MRK 493 & $5-30<$ & $-480,+60$ & 360,560 & 600 & 124000 & $+60 \pm 30$ & $360 \pm 50$ & 1.8 \\
\hline MRK 817 & $12-35$ & $-450,+300$ & 850,1200 & 140 & 14000 & $0,+130$ & $1550 \pm 100$ & 1.8 \\
\hline MRK 841 & $15-50$ & $-750,-150$ & 1070,1800 & 450 & 27400 & $-300 \pm 30$ & $1500 \pm 100$ & 2.1 \\
\hline NGC 3227 & $12-34$ & $-780,-300$ & 900,1550 & 350 & 12000 & $-300,300$ & $1500 \pm 100$ & 2.1 \\
\hline NGC 4253 & $5-25<$ & $-630,-90$ & 280,850 & 500 & 69500 & $-90,-30$ & $550 \pm 50$ & 2.0 \\
\hline PG 1116 & $8-30<$ & $-450,0$ & 1100,1800 & 500 & 15800 & $0,+90$ & $1400 \pm 250$ & 2.2 \\
\hline PG 1211 & $8-30$ & $-660,0$ & 540,1100 & 600 & 67400 & $+90 \pm 30$ & $600 \pm 300$ & 1.9 \\
\hline
\end{tabular}

fit in Fig. 9b is also reasonable. From these fitting tests (without any constraints for the disk parameters) we are able only to give rough estimates of the disk parameters (see Table 3 ). We should mention here that for lines with the smallest asymmetry it was more difficult to estimate the parameters. Consequently, the estimated range of the parameters is higher (see Table 3, e.g. Mrk 110).

\section{Results and discussion}

As one can see from Fig. 9, the line profiles can be well fitted with the two-component model, but some of the parameters (e.g. the emissivity index, the inclination, the inner and outer radii) are not constrained. It is therefore not possible to find a unique solution for the model. For this, one should arbitrarily constrain at least one of the disk parameters ${ }^{5}$. However, it is obvious that in the AGNs of the sample, the shape of the line wings indicates radial motion, which may be caused by a disk-like geometry. In any case, the fitting tests described above allow us to obtain rough estimates of the kinematical parameters of the two-component model (Table 3). In Table 3 we present the estimated range of inclinations (i) and the minimal emissivity index $\left(p^{\mathrm{min}}\right)$, minimal and maximal value for shifts and widths of the Gaussian broadening term from the disk $\left(z_{1}^{\min , \max }, W_{1}^{\min , \max }\right)$, the shifts and widths of the Gaussian component $\left(Z_{\mathrm{G}}, W_{\mathrm{G}}\right)$, and estimates for the minimal inner radius $\left(R_{\mathrm{inn}}^{\min }\right)$ and maximal outer radius $\left(R_{\mathrm{out}}^{\mathrm{max}}\right)$.

Concerning the disk we can point out the following: (i) the maximal outer radius is in the range $10^{4}$ to $10^{5} R_{\mathrm{g}}$. (ii) The local random velocities in the disk are different from object to object and they are in the broad range from $\sim 300 \mathrm{~km} \mathrm{~s}^{-1}$

\footnotetext{
${ }^{5}$ E.g. the dimensions of the BLR obtained from reverberation studies might be used as a constraint.
}

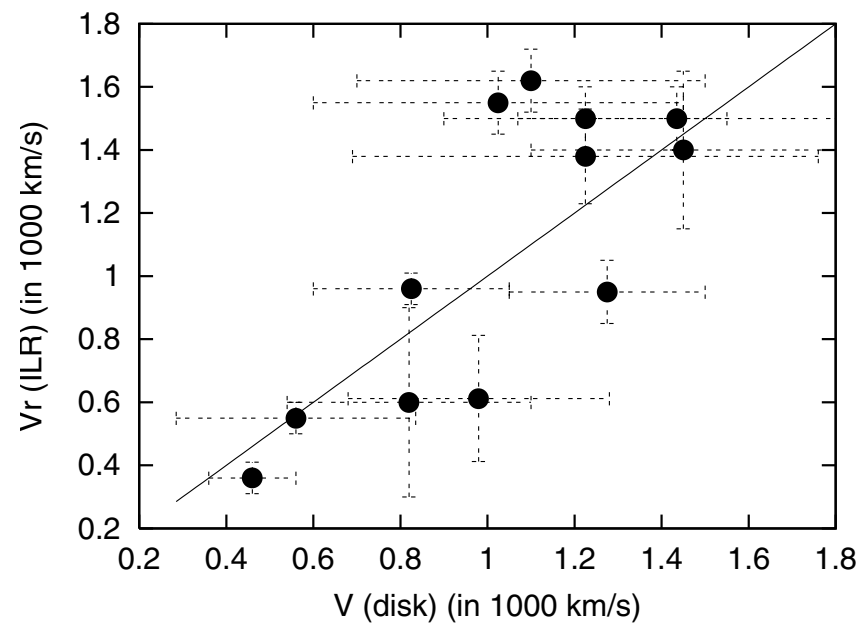

Fig. 10. The random velocities of a spherical region (ILR) as a function of the local random disk velocities. The dashed line represents the function $V_{\mathrm{ILR}}=V_{\text {Disk }} \mathrm{km} \mathrm{s}^{-1}$.

to $1700 \mathrm{~km} \mathrm{~s}^{-1}$; so are the local redshifts $\left(z_{1} \sim-780\right.$ to $+450 \mathrm{~km} \mathrm{~s}^{-1}$ ). (iii) The minimal inner radius of the emitting disk is in the range from $\sim 100$ to $600 R_{\mathrm{g}}$. (iv) The inclinations are $5^{\circ} \leq i<50^{\circ}$, and in about half of the AGNs $i<30^{\circ}$. Such values of the inclinations support the idea that we more frequently observe the Sy 1 type of AGNs at face-on inclinations (Rokaki \& Boisson 1999).

Concerning the spherical emission region we can point out that: i) the redshifts are within $300 \mathrm{~km} \mathrm{~s}^{-1}$ of the cosmological value; (ii) the random velocities in this region are also different for different objects, they are in the range from $\sim 400$ to $1600 \mathrm{~km} \mathrm{~s}^{-1}$. 
Particularly, it is interesting to see the correlation between local broadening in the two regions presented in Fig. 10. The local random velocities in the disk (averaged value from Table 3) are well correlated with those of the spherical region. This can be explained if this region originates from an accretion disk wind, which is created through several disturbances in the disk capable of producing shocks (e.g. Bondi-Hoyl flow, stellar wind-wind collision, and turbulences). Recently, Fromerth \& Melia (2001) described a scenario for the formation of a part of the BLR caused by shocks in the accretion disk; this may also create an ILR.

We should mention that besides a disk (or a disk-like region) or spiral shock waves within a disk (Chen et al. 1989; Chen \& Halpern 1989), other geometries may cause the same kinds of substructure in the line profiles: i) emission from the oppositely-directed sides of a bipolar outflow (Zheng et al. 1990, 1991); ii) emission from a spherical system of clouds in randomly inclined Keplerian orbits illuminated anisotropically from the center (Goad \& Wanders 1996); and iii) emission from a binary black hole system (Gaskell 1983, 1996). In any case one should consider a two-component model with an ILR contributing to the broad line cores and one additional emitting region contributing to the broad line wings. Recent investigations (see e.g. Wang et al. 2003; Eracleous \& Halpern 2003) have shown that the disk geometry for VBLR may be accepted as a reality. Moreover, Eracleous \& Halpern (2003) found that the disk emission is more successful not only in explaining double-peaked line profiles but also in interpreting the other spectroscopic properties of AGNs presenting these double-peaked Balmer lines.

\section{Conclusions}

We observed 12 AGNs with INT in order to obtain highresolution spectra of the $\mathrm{H} \beta$ and $\mathrm{H} \alpha$ lines that can be used for modeling BLRs. First we applied Gaussian analysis to the complex $\mathrm{H} \beta$ and $\mathrm{H} \alpha$ lines, from which we can conclude: (i) both the BLR and the NLR are complex; (ii) the [OIII] narrow lines can be fitted satisfactory only with two Gaussians, one shifted toward blue, which may indicate the existence of an outflow; (iii) the different parameters of these two Gaussians indicate that they originate in two NLR regions having different physical properties, (iv) the broad lines also show a complex structure, and they can be decomposed into three broad Gaussians one red-, one blue-shifted and one at the systemic redshift.

We adopt a two-component model, which comprises a VBLR and an ILR. We identify the VBLR with an accretion disk which contributes to the line wings. The cores of the lines originate in the ILR which is assumed to have a spherical geometry. This two-component model has been applied to the observed line profiles and we can conclude that: (i) the model can very well fit the observed line profiles, but it is very hard to obtain the disk parameters without imposing at least one constraint because of the large number of parameters and the lack of two peaks in the line profiles. They can be only roughly estimated using fitting tests (see Table 3). (ii) The random velocities in the spherical emission region and the random velocities in the disk are similar. This indicates that these two regions are linked through a common process, such as a wind produced by the disk.

To find constraints for the model parameters further investigations are needed.

Acknowledgements. The work was supported by the Ministry of Science, Technologies and Development of Serbia through the project "Astrophysical Spectroscopy of Extragalactic Objects" (L.C.P., E.B., and D.I.), the IAC through the project P6/88 "Relativistic and Theoretical Astrophysics" (E.M. and L.C.P.) and the Alexander von Humboldt Foundation through the program for foreign scholars (L.Č.P.). L.Č.P. \& E.B. thank to Institute for Astrophysics Canarias for the hospitality before and after observations. Also, we would like to thank the anonymous referee for very useful comments.

\section{References}

Arribas, S., Mediavilla, E., Garcia-Lorenzo, B., \& del Burgo, C. 1997, ApJ, 490, 227

Bennert, N., Falcke, H., Schulz, H., Wilson, A. S., \& Wills, B. J. 2002, ApJ, 574, 105

Chen, K., \& Halpern, J. P. 1989, ApJ, 344, 115

Chen, K., Halpern, J. P., \& Filippenko, A. V. 1989, ApJ, 339, 742

Corbin, M. R., \& Boroson, T. A. 1996, ApJS, 107, 69

Dopita, M. A., Bicknell, G. V., Sutherland, R. S., \& Saxton, C. J. 2003, Rev. Mex. Astron. Astrofis., 15, 323

Dumont, A. M., \& Collin-Souffrin, S. 1990, A\&AS, 83, 71

Eracleous, M. 2003, AGN Physics with the Sloan Digital Sky Survey (ASP Conf. Ser.), will appear 2004 [arXiv: astro-ph/0310649]

Eracleous, M., \& Halpern, J. P. 1994, ApJS, 90, 1

Eracleous, M., \& Halpern, J. P. 2003, ApJ, 599, 886

Fromerth, M. J., \& Melia, F. 2001, ApJ, 549, 205

Gaskell, C. M. 1982, ApJ, 263, 79

Gaskell, C. M. 1983, Proc. 24th Liege Intern. Ap. Colloquium, Univ. de Liege, Liege, 473

Gaskell, C. M. 1996, ApJ, 464, L107

Goad, M., \& Wanders, I. 1996, ApJ, 469, 113

Halpern, J. P., \& Oke, J. 1987, ApJ, 312, 91

Halpern, J. P., Eracleous, M., Filippenko, A. V., \& Chen, K. 1996, ApJ, 464, 704

Ho, L. C., Rudnick, G., Rix, H.-W., et al. 2000, ApJ, 541, 120

Kollatschny, W. 2003, A\&A, 407, 461

Kollatschny, W., \& Bischoff, K. 2002, A\&A, 386, L19

Korista, K. T. 1992, ApJS, 79, 285

Lípari, S., Terlevich, R., \& Macchetto, F. 1993, ApJ, 406, 451

Livio, M., \& Xu, C. 1997, ApJ, 478, L63

Murray, N., \& Chiang, J. 1997, ApJ, 474, 91

Nandra, K., George, I. M., Mushotzky, R. F., Turner, T. J., \& Yaqoob, T. 1997, ApJ, 477, 602

Perez, E., Mediavilla, E., Penston, M. V., Tadhunter, C., \& Moles, M. 1988, MNRAS, 230, 353

Popović, L. Č. 2003, ApJ, 599, 140

Popović, L. Č., Stanić, N., Kubičela, A., \& Bon, E. 2001, A\&A, 367, 780

Popović, L. Č., Mediavlilla, E. G., Kubičela, A., \& Jovanović, P. 2002, A\&A, 390, 473

Popović, L. Č., Mediavlilla, E. G., Bon, E., Stanić, N., \& Kubičela, A. 2003, ApJ, 599, 185

Rodríguez-Ardila, A., Pastoriza, M. G., \& Bica, E. 1996, ApJ, 463, 522

Rokaki, E., \& Boisson, C. 1999, MNRAS, 307, 41 
Romano, P., Zwitter, T., Calvani, M., \& Sulentic, J. 1996, MNRAS, Sulentic, J. W., Marziani, P., \& Dultzin-Hacyan, D. 2000, ARA\&A, 279, 165

Shields, J. C., Rix, H.-W., McIntosh, D. H., et al. 2000, ApJ, 534, L27
Sigut, T. A. A., \& Pradhan, A. K. 2003, ApJS, 145, 15

Smak, J. 1981, Acta Astron., 31, 395

Storey, P. J., \& Zeippen, C. J. 2000, MNRAS, 312, 813

Storchi-Bergmann, T., Eracleous, M., Ruiz, M. T., et al. 1997, ApJ, 489, 87

Storchi-Bergmann, T., Nemmen, R., Eracleous, M., et al. 2003, ApJ, 598, 956

Strateva, I. V., Strauss, M. A., Hao, L., et al. 2003, AJ, 126, 1720 38,521

Véron-Cetty, M.-P., \& Véron, P. 2000, A Catalogue of Quasars and Active Galactic Nuclei, Sci. Rep., 19

Véron, P., Goncalves, A. C., \& Véron-Cetty, M.-P. 2002, A\&A, 384, 826

Wang, J.-M., Ho, L. C., \& Staubert, R. 2003, A\&A, 409, 887

Wiese, W. L., Smith, M. W., \& Glennon, B. M. 1966, Atomic Transition Probabilities: Hydrogen Through Neon, Vol. I (Washington DC: NSRDS)

Zamanov, R., Marziani, P., Sulentic, J. W., et al. 2002, ApJ, 576, L9

Sulentic, J. W., Marziani, P., Zwitter, T., Calvani, M., \& Zheng, W., Binette, L., \& Sulentic, J. W. 1990, ApJ, 365, 115

Dultzin-Hacyan, D. 1998, ApJ, 501, 54

Zheng, W., Veilleux, S., \& Grandi, S. A. 1991, ApJ, 381, 418 
L. Č. Popović et al.: Contribution of the disk emission to the broad emission lines in AGNs, Online Material $p 1$

\section{Online Material}


L. Č. Popović et al.: Contribution of the disk emission to the broad emission lines in AGNs, Online Material p 2

Table 1. The observed AGNs with the coordinates, redshift, central wavelength and exposition.

\begin{tabular}{cccccccc}
\hline \hline $\begin{array}{c}\text { Object } \\
\text { name }\end{array}$ & $\begin{array}{c}\mathrm{RA} \\
\mathrm{h} \mathrm{m} \mathrm{s}\end{array}$ & $\begin{array}{c}\text { Dec } \\
\mathrm{o}^{\prime \prime \prime}\end{array}$ & $z$ & $\begin{array}{c}\text { Central } \\
\text { wavelength }(\AA)\end{array}$ & $\begin{array}{c}\text { Date of } \\
\text { observation }\end{array}$ & $\begin{array}{c}\text { Number of } \\
\text { spectra }\end{array}$ & $\begin{array}{c}\text { Exposures } \\
\mathrm{s}\end{array}$ \\
\hline Mrk 1040 & $2: 28: 14.3$ & $+31: 18: 40.4$ & 0.016652 & 6742.3 & 24-Jan.-02 & 4 & 600 \\
& & & & 4901.6 & 25-Jan.-02 & 4 & 1400 \\
\hline 3C 120 & $4: 33: 11.2$ & $+05: 21: 27.4$ & 0.033010 & 6742.3 & 24-Jan.-02 & 4 & 450 \\
& & & & 5027.9 & 22-Jan.-02 & 3 & 900 \\
\hline NGC 3227 & $10: 23: 29.6$ & $+19: 52: 15.2$ & 0.003839 & 6742.3 & 24-Jan.-02 & 4 & 480 \\
& & & & 4899 & 25-Jan.-02 & 3 & 600 \\
\hline PG 1116+215 & $11: 19: 08.7$ & $+21: 19: 33.9$ & 0.175700 & 5718.5 & 23-Jan.-02 & 3 & 1200 \\
& & & & 7699.1 & 24-Jan.-02 & 3 & 500 \\
\hline NGC 4253 & $12: 18: 26.1$ & $+29: 48: 57.5$ & 0.012929 & 4475.6 & 23-Jan.-02 & 4 & 1200 \\
& & & & 6742.3 & 24-Jan.-02 & 8 & 460,500 \\
\hline Mrk 110 & $9: 25: 11.3$ & $+52: 17: 29.4$ & 0.035291 & 5027.9 & 22-Jan.-02 & 3 & 1200,1500 \\
& & & & 6742.3 & 24-Jan.-02 & 4 & 720 \\
\hline Mrk 141 & $10: 19: 10.0$ & $+63: 58: 36.6$ & 0.041673 & 6744.8 & 24-Jan.-02 & 4 & 800 \\
\hline 3C 273 & $12: 29: 08.7$ & $+02: 03: 46.7$ & 0.158339 & 5669.4 & 25-Jan.-02 & 2 & 550 \\
& & & & 7699.1 & 24-Jan.-02 & 3 & 500 \\
\hline Mrk 817 & $14: 36: 20.5$ & $+58: 48: 14.6$ & 0.031455 & 4904.3 & 25-Jan.-02 & 3 & 550 \\
& & & & 6742.3 & 24-Jan.-02 & 3 & 500 \\
\hline Mrk 493 & $15: 59: 09.6$ & $+35: 02: 21.1$ & 0.031328 & 6742.3 & 24-Jan.-02 & 3 & 360 \\
\hline Mrk 841 & $15: 04: 03.3$ & $+10: 26: 48.1$ & 0.036422 & 6742.3 & 24-Jan.-02 & 3 & 450 \\
& & & & 4901.6 & 25-Jan.-02 & 6 & 300 \\
\hline PG 1211+143 & $12: 14: 19.4$ & $+14: 03: 44.0$ & 0.080900 & 5027.9 & 22-Jan.-02 & 3 & 600 \\
& & & & 6742.3 & 24-Jan.-02 & 3 & 500 \\
\hline
\end{tabular}

